\title{
Reversal of type 1 diabetes using plant-based diet: A case study.
}

\author{
Biswaroop Roy Chowdhury* \\ Medical Nutritionist, Indo-Vietnam Medical Board, India
}

Accepted on May 14, 2019

\begin{abstract}
Type-1 diabetes is an autoimmune disease characterized by hyperglycemia, inability to produce insulin due to self-destruction of beta cells in the pancreas. The epidemic of type- 1 diabetes causes irreversible suffering like retinopathy, nephropathy, neuropathy, foot complications, high blood pressure, etc, and put patients on a life sentence with insulin. The common perception in medical science is that sugar levels cannot be normalized without the help of medication. However, in the present study we examined a type 1 patient by putting her on a diet plan with regular follow ups and studied all diabetes-related biochemical parameters. We were successfully able to eliminate her medication and insulin dependency.
\end{abstract}

\section{Introduction}

Diabetes is one of the most common metabolic disorders associated with many life-threatening complications which make the life of a diabetic person worse. Diabetes is of two types- type 1 diabetes and type 2 diabetes. Type 2 is the more common type of diabetes worldwide (90-95\%), marked by an increased blood sugar level, frequent urination and weight loss. The other is type 1 or gestational diabetes (5-10\%). In type 2 diabetes, impaired receptors do not respond to insulin, eventually leading to insulin resistance while in type 1 there is a deficiency of insulin [1].

According to a recent estimate of International diabetes federation, South East Asia region, in 2017, there were $72,946,400$ cases in India, 425 million cases in the world and 82 million in SEA (South East Asia) region, which is likely to go up to 151 million by 2045 [2]. According to an estimate drawn in 2015, India is housing about 97,700 children with type 1 diabetes mellitus (T1DM). Although type 1 is not as prevalent as type 2, it shows a trend of 3-5\% increase every year [3]. India itself records three new cases of T1DM/100,000 children aged between 0-14 yrs. Three sets of prevalence data show 17.93 cases/100,000 children in Karnataka, 3.2 cases/ 100,000 children in Chennai and 10.2 cases/100,000 children in Karnal (Haryana) [4]. Type 1 diabetes is a juvenile onset disorder which is characterized by pancreatic dysfunction due to autoimmune reaction of the body where beta cells continuously get destroyed, eventually leading to insulin deficiency [5]. One highly sensitive method of C-peptide determination has shown that beta cells can be detected even up to 40 years after the clinical onset of the disease. These findings help patients with advanced diseases, for example, it helps patients whose b-cell function was thought to have long ceased in getting benefit from interventions to preserve b-cell function or to prevent complications [6]. A lot of effort has been made to regenerate beta cells in vitro by providing growth factors and by reprogramming/trans differentiation of terminally differentiated cell types [7]. However, none of these approaches have proved to be successful in growing beta cells in vivo due to shortage of donors. The inefficient technique of $\beta$-cell generation and the difficulty of growing $\beta$-cell in adult humans sufficiently forces a patient to adhere to medication for his whole life. Change in food habits plays an important role in both type 1 and type 2 diabetes by modulating or completely restoring normal glucose levels, thereby either decreasing or eliminating the need of medicine/insulin [8].

\section{Case Presentation}

Here, we present a case of a 23-year-old female, weighing 60 $\mathrm{kg}$, who was diagnosed with type 1 diabetes at Fortis, Mohali on 28 Dec 2011. She had been on insulin (60 U/day) for eight years before coming to us. She complained about her vision and was diagnosed with glaucoma in both her eyes and retinal detachment in her left eye. Her eyes had been operated on thrice. She displayed a nephropathy condition as stones were also found in her kidney.

She had been taking basic intervention through our video [9] for five months (Aug 2018-Dec 2018), after which, she approached us using the Diabetes 72 app [10] in Jan 2019. She was put on intensive intervention, where she was monitored regularly by a six-member health team in Faridabad.

\section{Intervention}

For the initial five months, she had been following the videorecommended diet, which includes cooked food along with raw food. This diet is divided into breakfast, lunch and dinner. Breakfast includes four different types of fruits which weigh equal to body weight (in $\mathrm{kg}) \times 10=\ldots(\mathrm{gm})$. Lunch includes 
four types of raw vegetables which weigh equal to body weight (in $\mathrm{kg}) \times 5=\ldots(\mathrm{gm})$ along with a normal cooked meal. Dinner is calculated the same way as lunch. In addition to this, soaked nuts and sprouts are also a part of the diet and the quantity of these also depend on the patient's body weight $(\mathrm{kg}) \ldots(\mathrm{gm})$. Sunshine is also an integral part of the prescribed diet. Packed and refined food, nutritional supplements, non-steroidal antiinflammatory drugs (NSAIDs) and dinner at late hours are strictly denied.

After five months, she was recommended to follow a more restricted diet plan mainly consisting of fruits and raw vegetables. Dairy products and cooked food were completely eliminated during intensive intervention [8]. A regular monitoring of glucose (fasting and post prandial) was carried out during intervention.

\section{Biochemical parameters}

Before intervention: The patient had been suffering from type 1 diabetes for eight years as was diagnosed in Dec 2011. She was prescribed 28 units before breakfast and 22 units before dinner of Mixtard insulin, and 10 units before lunch of Actrapid. In addition to that, she was taking $1000 \mathrm{mg}$ of metformin per day. Her blood sugar level varied from $156 \pm 24$ $\mathrm{mg} / \mathrm{dl}$ with total insulin (60 Unit) and $159 \pm 5$ with $25 \mathrm{U}$ insulin. Her HbAlc was $9.3 \%$ in 2011 and mean plasma glucose was 197 at the time of diagnosis (Table 1).

Post intervention: The intervention was divided into two phases.

Basic intervention: Where cooked food was offered along with raw food (Aug 2018-Jan 2019).

Intensive intervention: Where no cooked food was given (4-6 Jan 2019).

Her dependency on insulin decreased gradually from $60 \mathrm{U}$ to $25 \mathrm{U}$ during basic the intervention phase, and eventually to nil during intensive intervention phase. Her HBA1c was 7.2\% post intervention. Her C-peptide level was 0.93 (0.81-3.85) ng/ml, mean plasma glucose was 160 and Glutamic acid decarboxylase (GAD) $7 \mathrm{U} / \mathrm{ml}$ (value $<30$ is considered negative) after dietary intervention (Table 1$)$.

Table 1. The biochemical parameters before and after intervention.

\begin{tabular}{|c|c|c|c|c|c|c|}
\hline Variables & Date & Insulin & C-peptide & GAD & HbA1c (\%) & Blood sugar in average $(\mathrm{mg} / \mathrm{dl})$ \\
\hline \multirow{3}{*}{ At the time of diagnosis (2011) } & \multirow{3}{*}{$(28.12 .11)$} & Mixtrad & \multirow{3}{*}{-} & \multirow{3}{*}{-} & \multirow{3}{*}{9.3} & \multirow{3}{*}{$160^{*}$} \\
\hline & & $28 U+22 U /$ day & & & & \\
\hline & & Actaprid $10 \mathrm{U} /$ day & & & & \\
\hline \multirow{2}{*}{ Basic intervention (2019) } & Till mid-August & $50 \mathrm{U}$ & \multirow{2}{*}{-} & \multirow{2}{*}{-} & \multirow{2}{*}{ - } & $156^{*}$ \\
\hline & From mid Aug to Dec & $25 U$ & & & & $159^{*}$ \\
\hline \multirow{3}{*}{ Intensive intervention (2019) } & 4th Jan & 0 & - & - & - & 195 \\
\hline & 5th Jan & 0 & - & - & - & 181 \\
\hline & 6th Jan & 0 & - & - & - & 176 \\
\hline \multirow{2}{*}{ At the time of preparation of manuscript } & \multirow[b]{2}{*}{-} & \multirow{2}{*}{0} & 0.93 & 7 & 7.2 & \multirow[b]{2}{*}{-} \\
\hline & & & $(19.2 .2019)$ & $(5.3 .2019)$ & (18.2.2019) & \\
\hline
\end{tabular}

Note: GAD = Glutamic Acid Decarboxylase, Parenthesis (-) represents the non-availability of data, ${ }^{*}$ Glucose levels with insulin therapy

\section{Discussion}

Type 1 diabetes has been neglected for a long time and its nationwide prevalence is not yet known. The growing number of type 1 cases is a cause of concern as its treatment is difficult in comparison to that of type 2 [11]. In this article, we show a comprehensive analysis by stating the pre and post diabetic condition of a proband severely affected with type 1 diabetes who approached us using the Diabetes 72 app in Jan 2019. Her HbAlc reading was close to 8 every time, which is on the higher side. Higher values of HBA1c are suggestive of diabetic complications [12] which are evident by her eye and kidney problem. Her insulin dependency reduced to less than half after a basic dietary intervention, and finally, to nil after intensive intervention. Originally, when she was diagnosed as diabetic, her insulin intake was 60U in 2011 per day, and it came down to $25 \mathrm{U}$ in 2018 per day owing to dietary intervention. Presently, she is no longer dependent on insulin. Her glucose levels are also in normal range without insulin (184 \pm 9$)$. Her GAD values are suggestive of the reversal of type-1 diabetes. She adheres to regular follow ups. Evidences of cases of type 1 diabetes due to disturbed lifestyle are increasing [13]. Dietary intervention is a great help to medical science in curbing type 1 diabetes in a higher percentage, without causing any side effects. The complete reversal of chronic type 1 diabetes through dietary intervention is a ray of hope to many patients who are in the trap of medicines.

\section{Conclusion}

Diet plays a major role in the reversal of autoimmune diseases like type 1 diabetes. If left untreated, diabetes type 1 can lead 
to severe complications. Type-1 diabetes was once thought to be irreversible and progressive after diagnosis, but evidences suggest it can be reversed by following an appropriate diet plan. Here, we successfully treated a patient suffering from type 1 diabetes with complications by prescribing a customized diet plan. We searched major literature databases like Research Gate, Pubmed and Google Scholar, but could not find any type 1 reversal study of this kind. Therefore, this is the first report of a diabetes type 1 reversal in the world as per best of our knowledge.

\section{Acknowledgement}

Author thanks Dynamic Memory Pvt. Ltd. for the financial support.

\section{References}

1. American Diabetes Association. Diagnosis and classification of diabetes mellitus diabetes. Diabetes Care 2014; 37: 81-90.

2. https://www.idf.org/our-network/regions-members/southeast-asia/members/94-india.html.

3. Kumar KM. Incidence trends for childhood type 1 diabetes in India Indian J Endocrinol Metab 2015; 19: 34-35.

4. Das AK. Type 1 diabetes in India: Overall insights. Indian J Endocrinol Metab 2015; 19: 31-33.

5. American Diabetes Association. Classification and Diagnosis of Diabetes. Diabetes Care 2016; 39: 13-22.

6. Wang L, Fraser N, Lovejoy BS, Faustman DL. Persistence of prolonged $\mathrm{C}$-peptide production in type 1 diabetes as measured with an ultrasensitive C-peptide assay. Diabetes Care 2012; 35: 465-470.

7. Tesemma SC, Getnet YA. Recent advance in diabetes therapy: Pancreatic beta cell regeneration approaches. Diabetes Manag 2016; 6: 108-118.

8. Chowdhary BR. Diabetes reversal by plant-based diet. J Metabol Synd 2017.

9. https://www.youtube.com/watch?v=BbGWZkBl5Hg.

10. https://biswaroop.com/diabetes72app.

11. http://www.biswaroop.com/wp-content/uploads/2016/12/ Last-Days-of-Diabetes-English-com.pdf

12. Zoungas S, Chalmers J, Ninomiya T, Li Q, Cooper ME, Colagiuri S, Fulcher G, De Galan BE, Harrap S, Hamet P, Heller S, MacMahon S, Marre M, Poulter N, Travert F, Patel A, Neal B, Woodward M; Advance Collaborative Group. Association of hbalc levels with vascular complications and death in patients with type 2 diabetes: Evidence of glycaemic thresholds. Diabetologia. 2012; 55: 636-643.

13. Piłaciński S, Zozulińska-Ziółkiewicz DA. Influence of lifestyle on the course of type 1 diabetes mellitus. Arch Med Sci 2014; 10: 124-134.

\section{*Correspondence to}

Biswaroop Roy Chowdhury

Medical Nutritionist

Indo-Vietnam Medical Board

India 